\title{
A virtually floating dual-mass accelerometer
}

Maarten De Bock, Pierre Woestyn, Johan Raman, Patrick De Baets, Pieter Rombouts

This document is an author's draft version submitted for publication Sensors And Actuators A: Pysical The actual version was published as [1].

\section{REFERENCES}

[1] M. De Bock, P. Woestyn, J. Raman, P. De Baets, and P. Rombouts, "A virtually floating dual-mass accelerometer," Sensors and Actuators A-Physical, vol. 194, pp. 140-148, May 1 2013. [Online]. Available: http://www.sciencedirect.com/science/article/pii/S0924424713000411 


\title{
A virtually floating dual-mass accelerometer
}

\author{
Maarten De Bock ${ }^{1, *}$, Pierre Woestyn $^{1}$, Johan Raman ${ }^{1}$, Patrick De Baets ${ }^{\mathrm{b}}$, Pieter Rombouts ${ }^{1}$ \\ ${ }^{a}$ Dept. Elect. and Inform. Systems, Faculty of Engineering and Architecture, UGhent, Sint-Pietersnieuwstraat 41, 9000 Ghent, Belgium \\ ${ }^{b}$ Dept. Mechanical Construction and Production, Faculty of Engineering and Architecture, UGhent, Technologiepark Zwijnaarde 903, 9052 Zwijnaarde, Belgium
}

\begin{abstract}
A novel microelectromechanical system (MEMS) for the measurement of acceleration is presented. It combines a new mechanical structure consisting of two masses with a novel readout principle. The differential-mode displacement of the two proof masses corresponds to the applied acceleration and is embedded in a force-feedback loop. A new readout circuit is presented to give a readout proportional to this differential-mode. In addition, this new readout circuit has a control mechanism to operate the commonmode displacement of this dual-mass structure at the pull-in point. This increases the mechanical and electrical sensitivity.

As a proof of concept, a prototype was designed for the complete system. The accuracy of the system is $4.1 \mu \mathrm{g} / \sqrt{\mathrm{Hz}}$, which corresponds to the mechanical noise floor of the mechanical structure.
\end{abstract}

Keywords: MEMS inertial sensors, force-feedback, capacitive readout circuits, negative spring, pull-in, voltage control, charge control

\section{Introduction}

Displacement MEMS accelerometers are based on a massdamper-spring system [1-3]. The displacement of the mass will be proportional to the acceleration with a factor $m / k=$ $1 /\left(2 \pi f_{0}\right)^{2}$ (with $m$ the mass, $k$ the spring constant and $f_{0}$ the resonance frequency). This displacement then leads to a change in capacitance of a sense capacitor between the proof mass and a fixed reference (stator). The ratio of this change in capacitance to the applied acceleration is the mechanical sensitivity. The electrical sensitivity of the electronic readout circuit is then the ratio of the readout voltage to the change in capacitance.The two main noise sources in this system are the Brownian noise associated with the damping in the mechanical system and the electronic noise in the readout circuit [4]. In most designs, the electronic noise is dominant [3]. To reduce the influence of the electronic noise, it's input referred contribution has to be minimized by increasing the mechanical sensitivity and/or the electrical sensitivity.

For a force-feedback accelerometer, the closed-loop bandwidth can be set independently from the mechanical resonance frequency $f_{0}$ [2]. Hence, the mechanical sensitivity can be increased by decreasing $f_{0}$, but there are practical limits to this. First, the mass is limited by the available die-space. Second, reliability issues related to stiction (during the manufacturing process) and shock revival requires sufficiently stiff springs. As a result, a trade-off between reliability and sensitivity of the MEMS inertial sensor needs to be considered [1].

For the readout with parallel-plate capacitors, a technique has been introduced in [5] to enhance the mechanical sensitivity under "powered conditions". It uses the electrostatic force

\footnotetext{
${ }^{*}$ Corresponding author

Email address: maarten.debock@elis. ugent.be (Maarten De Bock)
}

between the capacitor plates to create a negative spring which can then compensate the mechanical spring. By matching the magnitude of the negative (electrostatic) springs and the mechanical springs, it is possible to obtain a zero effective spring, which makes the proof mass "virtually floating". This virtually floating condition is marginally unstable due to a phenomenon called "pull-in" [1], but this problem is tackled by embedding the mass in a force-feedback loop. The new architecture presented in this paper, will also exploit the negative spring effect to increase the mechanical sensitivity.

The mechanical sensitivity can also be increased by decreasing the gap between the parallel-plate sense capacitors. However, the minimum producible gap size is limited by the MEMS technology. Techniques have been presented to reduce this minimum gap mechanically after production with a lever and lock approach [6]. However, this gives substantial overhead to the mechanical structure and possibly reliability problems with respect to shock survival. In this paper, we explore a new electrostatic actuation method to reduce the gap during operation with a differential setup of two proof masses.

For the capacitive readout of MEMS inertial sensors, typically a voltage step is applied to the readout capacitors. In this case, increasing the amplitude of the readout voltage step results in a better signal-to-noise ratio (SNR) of the overall readout circuit, because the signal increases while the circuit noise remains the same [7]. However, above some critical voltage applied to the parallel-plate capacitors, the electrostatic force will become too high and pull-in of the proof mass occurs (in open loop) [1]. As noted above, by applying sufficiently strong negative force-feedback, the stability of the complete system can be guaranteed, even though the mechanical structure is at the marginally stable pull-in state.

In the new system introduced in this paper, the voltage step is 


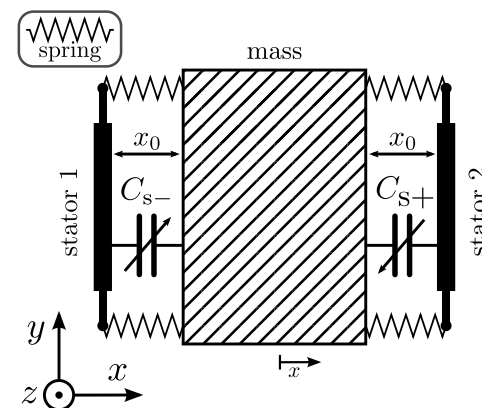

(a)

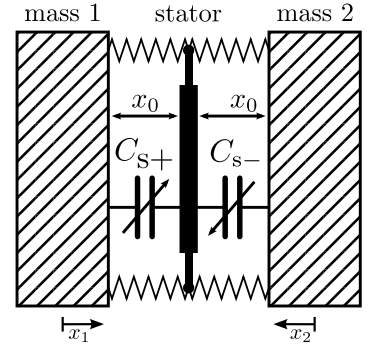

(b)
Figure 1: Schematic representation of a MEMS accelerometer: (a) conventional solution consisting of a single proof mass between two stator electrodes, (b) proposed solution consisting of one stator electrode in between 2 proof masses.

maximized toward the pull-in voltage to increase the electrical sensitivity. At the same time, operating at the pull-in distance brings the advantage that the mechanical springs are neutralized and that the sense capacitors are increased. Hence, also the mechanical sensitivity is increased.

The rest of this paper is divided in two main parts. The first part (Section 2) highlights the theoretical aspects of this new structure. The second part is a proof of concept for this new structure and covers the implementation (Section 3) and measurement results (Section 4) of a printed circuit board (PCB) implementation of the readout circuit for the new mechanical structure.

\section{A dual-mass accelerometer}

\subsection{New mechanical structure}

As a first step, consider a conventional 1-DoF MEMS accelerometer depicted in Fig. 1(a). It has a single proof mass which can move along the $\mathrm{X}$-axis and it is held back by springs. The displacement of the proof mass will be measured through a change in capacitance of a sense capacitor. On each side of the proof mass we have a sense capacitor, with one sense capacitor $C_{\mathrm{s}+}(x)$ increasing and the other $C_{\mathrm{s}-}(x)$ decreasing for a positive $\mathrm{X}$-displacement.

The idea of the new MEMS configuration is represented in Fig. 1(b) [8]. Compared to the conventional structure with one proof mass and two stator electrodes, the situation is now reversed: we only have a single stator electrode and a double proof mass with independent displacements $x_{1}$ (left mass) and $x_{2}$ (right mass) toward the stator. The sense capacitors are implemented as parallel-plate capacitors. As will be highlighted in Section 2.2, the capacitance of a parallel-plate capacitor is $C_{\mathrm{s}}(x)=C_{0} /\left(1-x / x_{0}\right)$. The displacement of the first mass is then read through $C_{\mathrm{s}+}=C_{0} /\left(1-x_{1} / x_{0}\right)$, while the displacement of the second mass is read through $C_{\mathrm{s}-}=C_{0} /\left(1-x_{2} / x_{0}\right)$.

Instead of working with $x_{1}$ and $x_{2}$, we consider a "normalized differential-mode displacement" $x_{\mathrm{dm}}=\left(x_{1}-x_{2}\right) / 2 x_{0}$ which indicates the displacement of both masses in the positive $\mathrm{x}$-direction, and a "normalized common-mode displacement" $x_{\mathrm{cm}}=\left(x_{1}+x_{2}\right) / 2 x_{0}$ which indicates the displacement of both

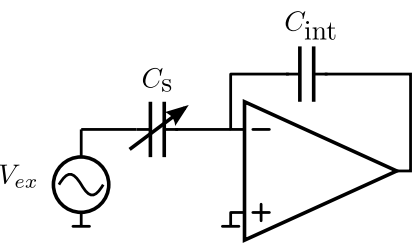

(a)

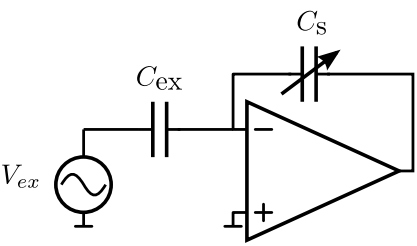

(b)
Figure 2: Readout circuits in (a) voltage control and (b) charge control.

masses toward the stator. Note that the differential displacement is related to the input force.

In a similar way as for the displacement, we can also define a "common-mode capacitance" $C_{\mathrm{cm}}$ and a "differential-mode capacitance" $C_{\mathrm{dm}}$ :

$$
\left\{\begin{array}{l}
C_{\mathrm{cm}}=\frac{C_{\mathrm{s}+}+C_{\mathrm{s}-}}{2}=C_{0} \frac{1-x_{\mathrm{cm}}}{\left(1-x_{\mathrm{cm}}\right)^{2}-x_{\mathrm{dm}}^{2}} \\
C_{\mathrm{dm}}=\frac{C_{\mathrm{s}+}-C_{\mathrm{s}-}}{2}=C_{0} \frac{x_{\mathrm{dm}}}{\left(1-x_{\mathrm{cm}}\right)^{2}-x_{\mathrm{dm}}^{2}}
\end{array}\right.
$$

To first order, the differential-mode capacitance relates to the differential-mode displacement and the common-mode capacitance relates to the common-mode displacement. Using the last equations, we find the ratio of the differential-mode and common-mode capacitance as:

$$
\frac{C_{\mathrm{dm}}}{C_{\mathrm{cm}}}=\frac{x_{\mathrm{dm}}}{1-x_{\mathrm{cm}}}
$$

This equation indicates one of the main features of this new structure: the sensitivity toward $x_{\mathrm{dm}}$ can be improved by increasing $x_{\mathrm{cm}}$. In others words, by moving both masses toward the stator, the sense capacitor gap is reduced which enhances the sensitivity. This will be done with electrostatic actuation through the parallel-plate sense capacitors. Moreover, reducing the capacitor gap with electrostatic actuation will also further increase the mechanical and electrical sensitivity, as will be discussed underneath (Section 2.2-2.4).

\subsection{Actuation and readout with parallel-plate capacitors}

The sense capacitor is implemented as a parallel-plate capacitor between a fixed plate (stator) and a moveable plate (mass) as shown in Fig. 3. The mass can move along the x-direction and is held back by springs and a corresponding spring force $F_{\mathrm{k}}=-k x$. The capacitor plates have a length $L_{\mathrm{f}}$ and a height $h$ (not shown in this cross Section view), while the nominal gap between the two plates is $x_{0}$. In practice, many fingers $\left(N_{\mathrm{f}}\right)$ are used in parallel. This leads to a total capacitance

$$
C_{s}(x)=\frac{C_{0}}{1-\frac{x}{x_{0}}}
$$

where we have introduced the nominal capacitance $C_{0}=$ $\varepsilon_{0} N_{\mathrm{f}} L_{\mathrm{f}} h / x_{0}$.

As noted above, we want to move the two proof masses toward the stator. For this, the same sense capacitors are used for electrostatic actuation. This actuation can be done by either voltage control or charge control. When a voltage or charge is 


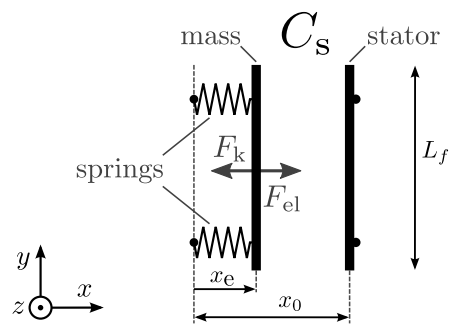

Figure 3: The parallel-plate capacitor $C_{\mathrm{s}}$ between a moveable mass and a fixed reference (stator). The mass is being held back by springs.

applied upon a capacitor, there will be an electrostatic force $F_{\mathrm{el}}$ between the two plates of the capacitor. This force will bring the moveable plate (mass) closer to the stator. As the mass is also being held back by a spring force $F_{\mathrm{k}}$, it will settle in a new equilibrium point $x_{\mathrm{e}}\left(0 \leq x_{\mathrm{e}}<x_{0}\right)$ where the forces cancel each other.

The characteristics of this equilibrium will be different for voltage or charge control. For voltage control, an excitation voltage is applied upon the sense capacitor $C_{s}$ (Fig. 2(a)). Changes in sense capacitance lead to a change in charge transferred onto the integration capacitor $C_{\text {int }}$. In [1] it is shown that voltage control is only stable if the voltage applied on $C_{\mathrm{s}}$ is below a certain pull-in voltage:

$$
V_{\mathrm{PI}}=\sqrt{\frac{8}{27} \frac{k x_{0}^{2}}{C_{0}}}
$$

If the voltage over the parallel-plate capacitor becomes higher than this pull-in voltage, the electrostatic force becomes too high to be compensated by the spring and the moveable capacitor plate clashes into the stator, which is the previously mentioned "pull-in" phenomenon. The corresponding xdisplacement for this pull-in voltage is the pull-in point which is located at $x_{\mathrm{PI}}=x_{0} / 3$. An important characteristic is that "net spring force" $k_{\text {net }}$ is reduced due to this electrostatic force:

$$
k_{\text {net }}=\frac{\delta F_{\text {net }}}{\delta x}=k-V^{2} \frac{C_{0}}{x_{0}^{2}} \frac{1}{\left(1-x_{\mathrm{e}}(V) / x_{0}\right)^{3}}
$$

Here, $x_{\mathrm{e}}$ is a function of the applied excitation voltage $V$. Increasing $V$ reduces $k_{\text {net }}$, while also increasing $x_{\mathrm{e}}$, with a limit at the pull-in point.

For charge control, a fixed charge is applied upon the sense capacitor (Fig. 2(b)). A change in sense capacitance is then read through a change in voltage over the sense capacitor. If we now calculate the net spring constant, it can be shown that $k_{\text {net }}=k$, independent of the applied charge. This shows that applying a constant charge leads to a solution that is always stable [9-11]. Compared to voltage control, charge control does not result in a pull-in point, but there is also no negative spring effect.

\subsection{Differential-mode stabilization}

To control the 2 degrees of freedom ( $x_{\mathrm{dm}}$ and $x_{\mathrm{cm}}$ ), we will set up two control loops to stabilize both proof masses. As $x_{\mathrm{dm}}$ corresponds to the applied acceleration, we will embed the

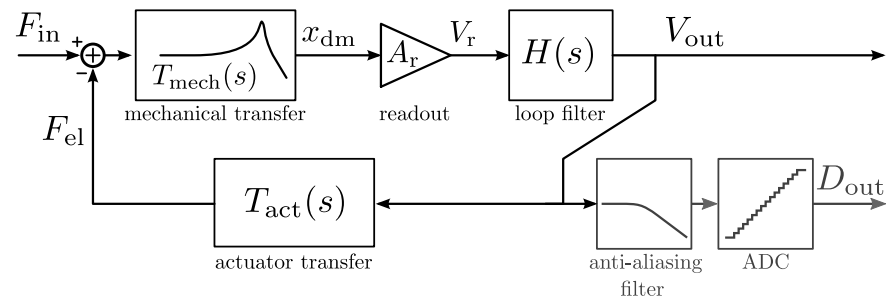

Figure 4: The force-feedback loop for the differential-mode.

differential-mode in a force-feedback loop, as in a conventional mono-mass dual-stator force-feedback accelerometer [1, 12].

Fig. 4 shows the resulting force-feedback loop. The input to this control loop is a force $F_{\text {in }}=m \cdot a_{\text {in }}$. The mechanical structure $\left(T_{\text {mech }}(s)\right)$ will transfer this input force into the differential displacement $x_{\mathrm{dm}}$ :

$$
T_{\text {mech }}(s)=\frac{x_{\mathrm{dm}}}{F_{\text {in }}}=\frac{1}{x_{0}} \frac{1 / k_{\text {net }}}{1+\frac{b}{k_{\text {net }}} s+\frac{m}{k_{\text {net }}} s^{2}}
$$

The static sensitivity of the resonator toward the input force is related to $\sim 1 / k_{\text {net }}$. Hence, reducing $k_{\text {net }}$ will increase the mechanical sensitivity. Since we want to increase the sensitivity of the differential-mode, we want the differential mode to have a negative spring effect. The readout of the differential mode of the proof masses must therefore be operated in voltage control. After applying an additional electric filter $H(s)$, the output of this filter $V_{\text {out }}$ is then fed back toward the input of the loop through an actuation circuit. The analog output voltage $V_{\text {out }}$ can be digitized with an analog-to-digital converter (ADC) to give the digital output $D_{\text {out }}$.

The force-feedback differential-mode stabilization can also be extended to a $\Sigma \Delta$-loop, which has become increasingly popular e.g. [13-15]. For this, a quantizer is added after the loop filter, before applying the digital feedback. With a proper designed loop filter [16-18], the added quantization noise can be made negligible and the performance will be further enhanced.

\subsection{Common-mode stabilization}

Because we actively maintain $x_{\mathrm{dm}} \approx 0$ with force-feedback, only $x_{\mathrm{cm}}$ remains to be controlled. If we now apply the same voltage $V$ (measured relative to the common stator) to both proof masses, an identical electrostatic force will occur and both will move over the same distance toward the center. It is clear that the system basically behaves in the same way as a single mass which forms a parallel-plate capacitor with the stator electrode.

Note that increasing $x_{\mathrm{cm}}$ will lead to an improved electrical sensitivity, as shown in Eq. (2), but that $x_{\mathrm{cm}}$ contains no information about the acceleration. Therefore, the negative spring effect, is of no importance for the common-mode stabilization. As such, we will use charge control for the common-mode stabilization, as it is inherently stable (Section 2.2).

We now need a readout circuit that combines charge control for $C_{\mathrm{cm}}$ with voltage control for $C_{\mathrm{dm}}$. This is possible, by applying a constant charge to the parallel combination of both sense capacitors. This way, the common-mode capacitor $C_{\mathrm{cm}}$, 


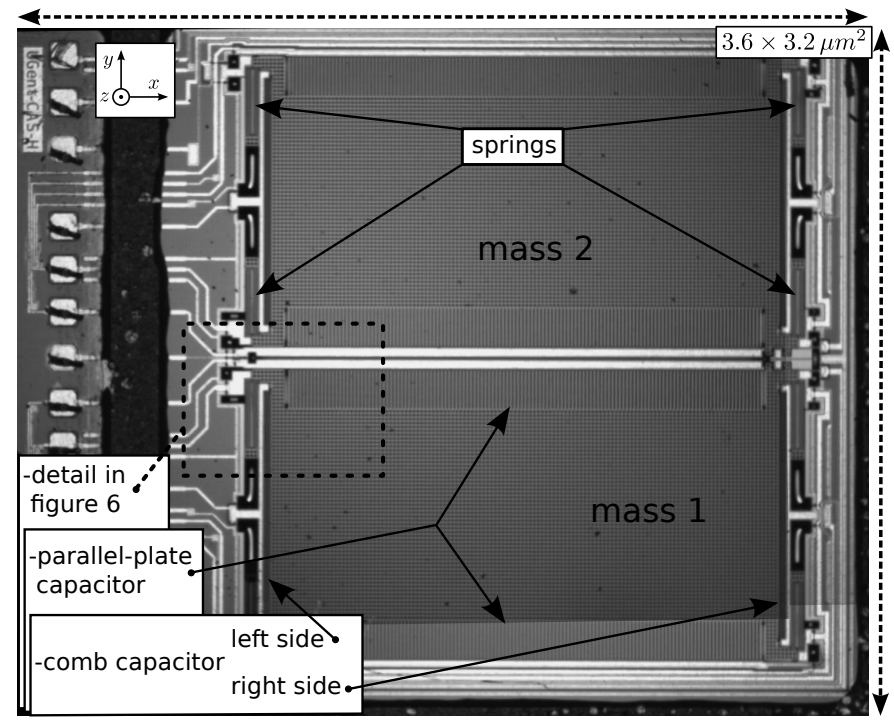

Figure 5: Microscope photograph of the fabricated dual-mass MEMS structure after topcap removal.

which corresponds to $x_{\mathrm{cm}}$, is certainly operated in charge control (Fig. 2(b)). However, due to the differential arrangement of the sense capacitors and due to the fact that both capacitors can still transfer charge between them, the differential-mode capacitor $C_{\mathrm{dm}}$ (and corresponding $x_{\mathrm{dm}}$ ) is operated in voltage control. The resulting pull-in point $x_{\mathrm{PI}}$ and voltage $V_{\mathrm{PI}}$ are exactly the same as for a single parallel-plate capacitor operated with voltage control (Fig. 2(a)). In this case, we can write the ratio of the net spring constant for the differential-mode to the nominal spring constant $k$ as:

$$
\frac{k_{\mathrm{net}}}{k}=\frac{1-3 x_{\mathrm{cm}}}{1-x_{\mathrm{cm}}}
$$

which becomes zero at exactly $x_{\mathrm{cm}}=1 / 3$, giving a virtually floating mass for the differential-mode.

An example of a readout circuit that combines charge control for the common-mode with a voltage controlled differentialmode readout will be presented in Section 3.2.

\section{Implemented prototype}

\subsection{Fabricated MEMS structure}

The mechanical structures are fabricated in a SurfaceMicromachined MEMS process for inertial sensors. The minimum feature size is $2 \mu \mathrm{m}$ and the device layer thickness is $15 \mu \mathrm{m}$. The dies are assembled in a standard available PLCC64 package, of which only the pins of one side are used to connect the 13 bondpads of the MEMS die.

A microscope picture of the fabricated structure (with removed topcap) is shown in Fig. 5. The bondpads for the various electrodes are located on the left side, while the masses are arranged above each other on the right with movement along the x-axis. Also each mass has it's own stator electrode (instead of a common stator as in Fig. 1(b)). The parallel-plate sense capacitors are placed at the top and bottom side of each mass.

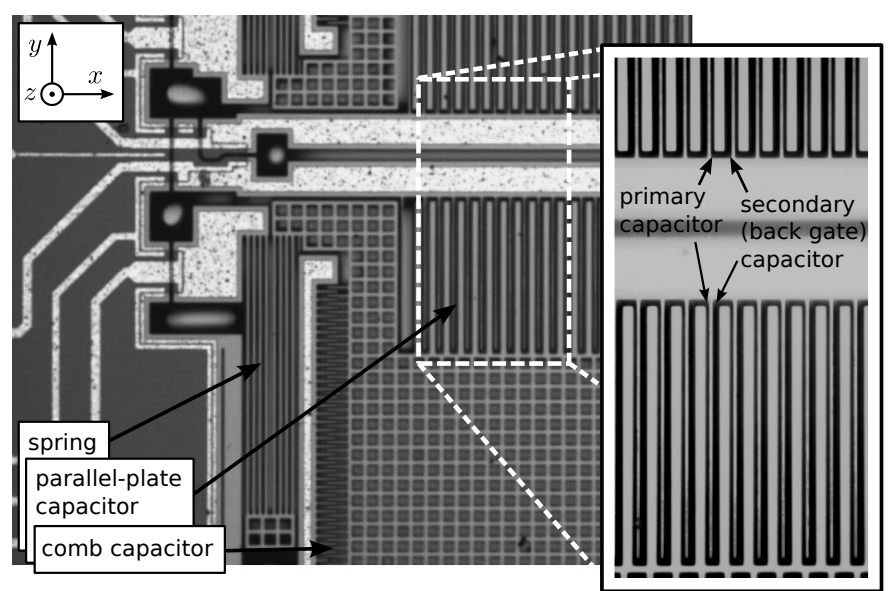

Figure 6: Microscope photograph showing a detail of the fabricated MEMS structure after topcap removal (with extra magnification of the parallel-plate capacitor).

Additional comb capacitors are placed at the left and right side of the masses. A folded-beam spring [19] is placed at the 4 corners of each mass.

Fig. 6 shows a close-up with details of the bottom mass: the spring attached at the corner, the comb capacitor on the left side and the parallel-plate capacitor at the top side. The picture also shows an extra close-up on the parallel-plate capacitors. In particular, it also shows that every capacitor finger has an (undesired) secondary capacitor at the back-gate.

The design parameters of the MEMS structure are summarized in Table 1. The proof masses are as large as possible, within the limitations of the maximum die size. Note that the density of the mass is quite low, as Fig. 6 clearly shows the waffle pattern of the mass, needed for proper etching of the sacrificial layer to release the mass. Also the parallel-plate capacitors are chosen to be as large as possible, within the constraints of feasibility, and are implemented with the minimum gap of $2 \mu \mathrm{m}$. The gap to the back gate is chosen twice this minimum gap size. The back gate capacitor will slightly change the equations for voltage and charge control, but the general conclusions remain the same: pull-in at $x_{\mathrm{cm}} \approx 1 / 3$ for voltage control and no net spring effect and increased stability for charge control. The resonance frequency of the structure is chosen such that the maximum pull-in voltage (within process deviations) is lower then $5 \mathrm{~V}$. To protect the MEMS structure, stoppers are provided which limit the displacements at $x_{i}=1.8 \mu \mathrm{m}$.

The damping force will be dominated by film squeeze effects [20]. Only the low frequency component of the film squeeze damping is important here, since the film-squeeze cut-off frequency is much higher than $f_{0}$. For this incompressible gas condition, the damping coefficient is

$$
b=\mu_{\mathrm{eff}} N L_{\mathrm{f}} \frac{h^{3}}{x_{0}^{3}} \beta(\eta)
$$

where $\beta(\eta)$ is function of the aspect ratio $\eta=h / L_{\mathrm{f}}$ [20] and $\mu_{\text {eff }}$ is the effective viscosity at the encapsulated air pressure. The parameters $h, L_{\mathrm{f}}$ and $N_{\mathrm{f}}$ describe the parallel-plate capacitor 


\begin{tabular}{|l|l|}
\hline Parameter & Value \\
\hline \hline Process & XFAB XM-SC XM20A17 \\
\hline Die size & $3600 \mu \mathrm{m} \times 3200 \mu \mathrm{m}$ \\
\hline Single mass size & $1954 \mu \mathrm{m} \times 1114 \mu \mathrm{m}$ \\
\hline Single mass: $m$ & $45.3 \mu \mathrm{g}$ \\
\hline Mass thickness: $h$ & $15 \mu \mathrm{m}$ \\
\hline Nominal gap: $x_{0}$ & $2 \mu \mathrm{m}$ \\
\hline Damping factor & \\
$\quad$ Nominal/zero position: $b_{\text {nom }}$ & $0.245(\mathrm{mNs}) / \mathrm{m}$ \\
$\quad$ Pull-in position: $b_{\mathrm{PI}}$ & $0.431(\mathrm{mNs}) / \mathrm{m}$ \\
\hline Spring constant: $k$ & $26.5 \mathrm{~N} / \mathrm{m}$ \\
\hline Parallel-plate capacitor: & \\
Primary capacitor: $C_{\mathrm{s}}$ & $2.56 \mathrm{pF}$ \\
Back gate capacitor: $C_{\mathrm{bg}}$ & $1.26 \mathrm{pF}$ \\
\hline Comb capacitor: $C_{\mathrm{cmb}}$ & $174 \mathrm{fF}$ \\
\hline Resonance frequency: $f_{0}$ & $3.85 \mathrm{kHz}$ \\
\hline Pull-in voltage: $V_{\mathrm{PI}}$ & $3.66 \mathrm{~V}$ \\
\hline
\end{tabular}

Table 1: The parameters of the MEMS structure.

as expressed before in Eq. (3). Border effects are included by introducing the effective dimensions as in [21] .

\subsection{Readout circuit}

The electronic readout circuit is implemented on a PCB for experimenting and debugging and is shown in Fig. 7. The dual charge integrator, combined with the common-mode feedback amplifier, is a variant of the readout circuit proposed in [13]. However, there are some important differences. First, sinusoidal excitation is used in combination with a multiplier for demodulation. Second, the excitation voltage is applied through the excitation capacitors $\left(C_{\mathrm{ex} 1}, C_{\mathrm{ex} 2}\right)$, while the common-mode feedback is applied through the sense capacitors $\left(C_{\mathrm{s}+}, C_{\mathrm{s}-}\right)$. For the implementation of the PCB prototype, a combination of 2 single ended charge integrators is preferred and as a result, output common-mode feedback is used.

\subsubsection{Charge control common-mode stabilization}

Let us first assume that the differential-mode displacement is zero. The common-mode regulating part of the readout circuit can then easily be understood through an equivalent circuit shown in Fig. 8. Here, the dual charge integrator and the common-mode feedback amplifier are combined and drawn as one overall gain stage $A_{\mathrm{CML}}$. Note the similarity between Fig. 8 and 2(b). This shows that the common-mode regulating control loop performs charge control on the common-mode capacitor $C_{\mathrm{cm}}$ (as noted in Section 2.4), which leaves the differentialmode still open for voltage control. The sinusoidal excitation voltage $\left(V_{\mathrm{ex}}=A_{\mathrm{ex}} \sin \left(2 \pi f_{\mathrm{ex}} t\right)\right)$ is transferred into an excitation charge $Q_{\mathrm{ex}}=V_{\mathrm{ex}} C_{\mathrm{ex}}$. If $A_{\mathrm{CML}}$ is sufficiently high at $f=f_{\mathrm{ex}}$, we can write :

$$
V_{\mathrm{cmfb}}=-\frac{Q_{\mathrm{ex}}}{C_{\mathrm{cm}}}=-\frac{C_{\mathrm{ex}}}{C_{\mathrm{cm}}} V_{\mathrm{ex}}
$$

We will now calculate the electrostatic force $F_{\text {el }}$ between the

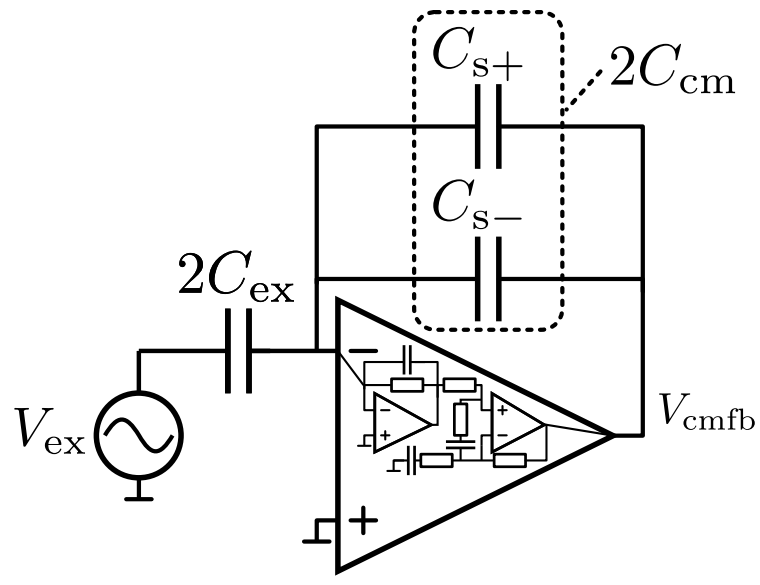

Figure 8: Equivalent circuit of the common-mode charge control. The charge integrator and common-mode feedback amplifier are here drawn as a single gain stage $A_{\mathrm{CML}}$.

plates of this common-mode capacitor. As the charge applied on $C_{\mathrm{cm}}$ is $Q_{\mathrm{ex}}$, we can write for the electrostatic force $F_{\mathrm{el}}$ :

$$
\begin{aligned}
F_{\mathrm{el}} & =-\frac{1}{2} Q_{\mathrm{ex}}^{2} \frac{d\left(1 / C_{\mathrm{cm}}\right)}{d x_{\mathrm{cm}}} \\
& =\frac{1}{2} \frac{C_{\mathrm{ex}}^{2} A_{\mathrm{ex}}^{2}}{C_{0}}\left(\frac{1-\cos \left(2 \cdot 2 \pi f_{\mathrm{ex}} t\right)}{2}\right)
\end{aligned}
$$

As this electrostatic force depends quadratically on $Q_{\mathrm{ex}}$, it actually performs a downconversion from $f_{\text {ex }}$ to DC. This electrostatic force is transferred by the mechanical transfer function $T_{\text {mech }}(s)$ into a common-mode displacement $x_{\mathrm{cm}}$. If the bandwidth of $T_{\text {mech }}(s)$ is substantially smaller than $2 f_{\text {ex }}$, we find:

$$
x_{\mathrm{cm}}=\frac{1}{2} \frac{C_{\mathrm{ex}}^{2}}{C_{0}} \frac{A_{\mathrm{ex}}^{2}}{2} \frac{1}{k}
$$

We see that increasing the excitation voltage amplitude $A_{\text {ex }}$ will increase $x_{\mathrm{cm}}$. The common-mode feedback amplifier is implemented as a non-inverting amplifier, with low frequency gain of $\left(R_{1}+R_{2}\right) / R_{1}=100$. For stability reasons, the gain-bandwidth product (GBWP) of the amplifier was reduced by adding resistor $R_{3}$. To limit the influence of the offset voltage of the opamp, large capacitors $C_{1}$ and $C_{3}$ are added, which reduce the DC-gain to 1 . The resistors $R_{\mathrm{fb} 1,2}$ are added to the charge integrators to have a stable output for DC signals and are large enough so that we can neglect them at the frequency of interest $\left(f=f_{\mathrm{ex}}\right)$.

\subsubsection{Differential-mode readout}

The differential output voltage $V_{\text {diff }}$ is operated voltage control and is determined with an instrumentation amplifier. Using Eq. (7), we can write:

$$
\begin{aligned}
V_{\mathrm{diff}} & =-\left(C_{s+}-C_{s-}\right) \frac{V_{\mathrm{cmfb}}}{C_{\mathrm{int}}} G_{\mathrm{d}} \\
& =\frac{C_{s+}-C_{s-}}{C_{s+}+C_{s-}}\left(2 \frac{C_{\mathrm{ex}}}{C_{\mathrm{int}}} V_{\mathrm{ex}}\right) G_{\mathrm{d}}
\end{aligned}
$$

This can be simplified using Eq. (1) and we find:

$$
V_{\mathrm{diff}}=\frac{x_{\mathrm{dm}}}{1-x_{\mathrm{cm}}}\left(2 \frac{C_{\mathrm{ex}}}{C_{\mathrm{int}}}\right) G_{\mathrm{d}} A_{\mathrm{ex}} \sin \left(2 \pi f_{\mathrm{ex}} t\right)
$$




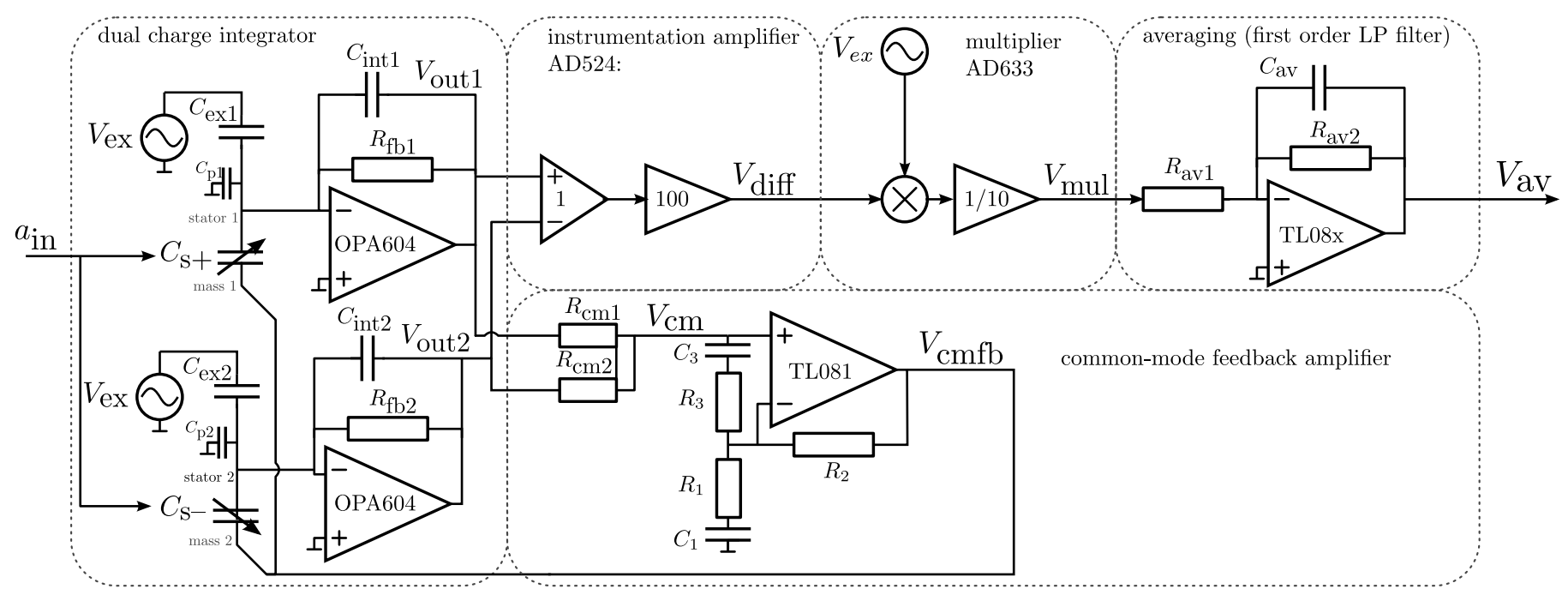

Figure 7: Complete open loop readout circuit with charge control common-mode stabilization.

This is then demodulated with a voltage multiplier and low-pass filtered, giving $V_{\mathrm{av}}$ :

$$
V_{\mathrm{av}}=G_{\mathrm{av}} G_{\mathrm{m}} G_{\mathrm{d}} \frac{x_{\mathrm{dm}}}{1-x_{\mathrm{cm}}}\left(2 \frac{C_{\mathrm{ex}}}{C_{\mathrm{int}}}\right) A_{\mathrm{ex}}^{2}\left(\frac{1}{2}\right)
$$

The carrier frequency has to be substantially higher than the resonance frequency of the MEMS structure $\left(f_{0}=3.85 \mathrm{kHz}\right)$, but it is also limited by the bandwidth of the instrumentation amplifier and the common-mode feedback amplifier. As a result, the carrier frequency was chosen at $50 \mathrm{kHz}$. The capacitors $C_{\text {ex }}$ and $C_{\text {int }}$ are chosen to be $4.7 \mathrm{pF}$, close to the actual sense capacitance.

\subsubsection{Noise analysis}

Due to the high gain of the instrumentation amplifier, the noise contributed by the charge integrators will be the dominant noise source for the electrical noise. The charge integrators are implemented with an OPA604 opamp (input referred noise: $V_{\mathrm{n}, \mathrm{op}}=10 \mathrm{nV} / \sqrt{\mathrm{Hz}}$ ). The total noise $V_{\mathrm{n} \text {,tot }}$ at the input of the instrumentation amplifier can be simplified to

$$
V_{\mathrm{n}, \text { tot }}=\left(1+\frac{C_{\mathrm{ex}}+C_{\mathrm{cm}}+C_{\mathrm{p}}}{C_{\mathrm{int}}}\right) \cdot \sqrt{2} \cdot 10 \frac{\mathrm{nV}}{\sqrt{\mathrm{Hz}}}
$$

where the factor $\sqrt{2}$ is added as both opamps' noise contributions are equal but uncorrelated. Since $C_{\mathrm{int}}=C_{\mathrm{ex}} \approx C_{\mathrm{cm}}$, this equation shows that the charge integrators are indeed the dominant electrical noise contributors when compared to the instrumentation amplifier (input referred noise of $7 \mathrm{nV} / \sqrt{\mathrm{Hz}}$ ).

\subsection{Force-feedback}

The force feedback is done by means of comb capacitors, located on the left and right hand side of the proof mass as shown in Fig. 9. If there are $N$ fingers of height $h$, overlapping over a distance $L_{0}+x$, and with a distance $D$ between the fingers, the total capacitance is:

$$
C_{\mathrm{cmb}}(x)=C_{0, \mathrm{cmb}}\left(1+\frac{x}{L_{0}}\right)
$$

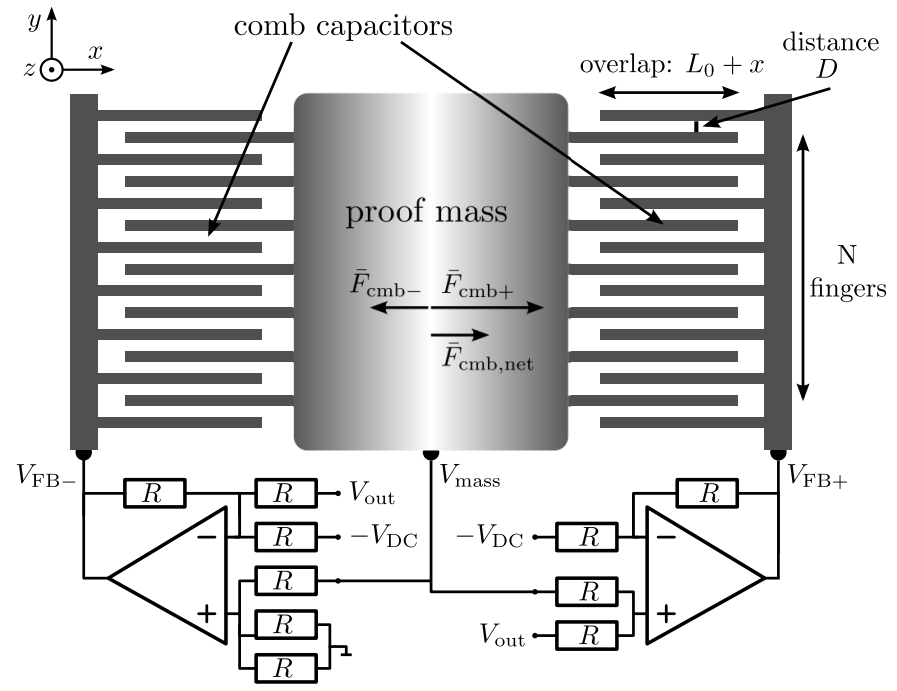

Figure 9: The proof mass with comb capacitors on both sides and the corresponding circuits to convert the actuation voltage $V_{\text {out }}$ into an actuation force $\bar{F}_{\text {cmb,net }}$.

The overlap in zero-position is written here as $L_{0}$ and the nominal capacitance at this zero position is $C_{0, \mathrm{cmb}}=\varepsilon_{0} N h L_{0, \mathrm{cmb}} / D$.

The force applied by these comb capacitors is linearized by adding a DC-bias voltage $\left(V_{\mathrm{DC}}\right)$ to the combs at both sides as shown by the circuits in Fig. 9. The resulting electrostatic force applied to the proof mass at voltage $V_{\text {mass }}$ is (with $V_{\text {out }}$ the feedback voltage):

$$
F_{\mathrm{cmb}, \text { net }}=F_{\mathrm{cmb}+}-F_{\mathrm{cmb}-}=\frac{1}{2} \frac{C_{0, \mathrm{cmb}}}{L_{0, \mathrm{cmb}}}\left(4 V_{\mathrm{DC}}\right) \cdot V_{\text {out }}
$$

As the mass electrode is connected to the output of the common-mode feedback amplifier (Fig. 7), it is a low impedance node that can easily drive this adder circuit. 


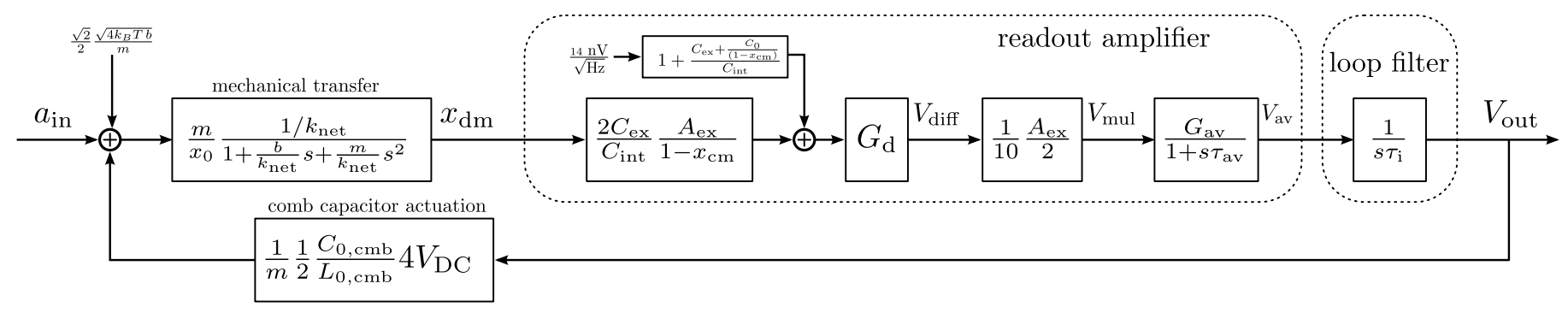

Figure 10: The system level diagram for the force-feedback loop of the implemented accelerometer.

\subsection{Complete system}

For the force-feedback loop, an additional loop filter is placed after the readout circuit before applying the feedback. The used loop filter is a simple integrator:

$$
H(s)=\frac{1}{s \tau_{\mathrm{i}}}
$$

The pole of the integrator will provide the dominant pole of the differential-mode force-feedback loop. Due to the $180^{\circ}$ phase shift in the mechanical part, the force-feedback will only be stable for a dominant pole sufficiently lower in frequency than the mechanical resonance frequency. The system bandwidth could be increased by using a more advanced filter with frequency compensation $[17,18]$, but this is beyond the scope of this prototype. The complete system level diagram for the differentialmode is then shown in Fig. 10. The input of the system is the external acceleration applied upon the proof masses. The proof masses convert this acceleration into a differential displacement $x_{\mathrm{dm}}$. This is converted into a voltage $V_{\mathrm{av}}$ by the readout amplifier, which is succeeded by the loop filter giving the output of the complete system, $V_{\text {out }}$. This signal is fed back to the input via the comb capacitor actuation circuit. The dominant electrical noise source is also drawn, which enters the loop after the dual charge integrator stage, given by equation (9). As the total loop gain will be very high for low frequencies, we can assume nullator at the input of the control loop. As such we can write for low frequencies:

$$
\frac{V_{\text {out }}}{a_{\text {in }}}=8 V_{\mathrm{DC}} \cdot \frac{C_{0, \mathrm{cmb}}}{L_{0, \mathrm{cmb}}} \cdot m
$$

As the excitation voltage $\left(A_{\mathrm{ex}}\right)$ is increased, the gain of the readout circuit will increase. First, in the dual charge integrator, by the factor $A_{\mathrm{ex}} /\left(1-x_{\mathrm{cm}}\right)$, where also an additional gain increase due to the gap reduction $\left(x_{\mathrm{cm}}\right)$ is apparent. The gain of the multiplier stage is also increased (factor $\frac{A_{\mathrm{ex}}}{20}$ ), but this additional gain will not reduce the input referred electrical noise. Second, the increased excitation voltage and corresponding increased $x_{\mathrm{cm}}$ will reduce the net spring constant (see Eq. (5)), and as such increases the mechanical gain. This will further reduce the input referred electrical noise. At a certain point, the Brownian noise will become the dominant noise source $a_{\mathrm{Br}}$. This mechanical noise is given by the formula [22]

$$
\frac{d a_{\mathrm{Br}}}{d f}=\frac{\sqrt{4 k_{B} T b}}{m}
$$

and is also added to the input of the system level diagram (Fig. 10).

For a damping factor $b=2.45 \cdot 10^{-4}(\mathrm{~N} \mathrm{~s}) / \mathrm{m}(\mathrm{Eq}$. (6)), this gives an acceleration spectral noise density of $4.5 \mu \mathrm{g} / \sqrt{\mathrm{Hz}}$ per mass. As the mechanical noise is uncorrelated for both proof masses, the corresponding noise for the normalized differential displacement $\left(x_{\mathrm{dm}}\right)$ has to be multiplied by a factor $\sqrt{2} / 2$ which gives a theoretical spectral noise density floor of $3.2 \mu \mathrm{g} / \sqrt{\mathrm{Hz}}$. Note that this noise density is the same for one proof mass with twice the mass, as we would have in a conventional mono-mass MEMS accelerometer structure. The mechanical noise source is also added to the system level diagram shown in Fig. 10. As the proof masses move up to the pull-in point, the damping factor will increase (Table 1), and the mechanical noise floor increases to $4.25 \mu \mathrm{g} / \sqrt{\mathrm{Hz}}$ at the pull-in point.

\section{Measurement results}

The mechanical amplitude transfer function (TF) of the MEMS structure is measured using the same readout circuit (Fig. 7). However, here the differential-mode is used in open loop without force-feedback, as the actuation circuit (Fig. 9) is used to apply a small sinusoidal force which is swept in frequency. The used excitation voltage $\left(A_{\mathrm{ex}}\right)$ is small, so the negative electrostatic spring effect is negligible. The measured amplitude transfer function of the applied actuation voltage toward the output voltage $V_{\text {av }}$ of the readout circuit is then shown in Fig. 11. It is normalized with the low frequency gain:

$$
\left|\frac{T F_{\text {meas }}(f)}{T F_{\text {meas }}(0)}\right|=\left|\frac{T F_{\text {mech }}(f)}{T F_{\text {mech }}(0)}\right|=\left|\frac{1}{1+\frac{b}{k} s+\frac{m}{k} s^{2}}\right|
$$

Also the normalized expected (designed) TF is shown in Fig. 11. It shows that the measured resonance frequency $f_{0}$ closely matches the expected value (given in Table 1), but the damping is slightly overestimated. This can be seen from a fitted transfer function (parameters $m / k$ and $b / k$ ). The ratio $m / k$ is only $2 \%$ higher, while the ratio $b / k$ is $20 \%$ lower than expected. If we assume that the values of the parameters $m$ and $k$ are exact (motivated by the matching of $f_{0}$ ), we can conclude that the damping factor is slightly overestimated by Eq. (6).

The mechanical TF is also measured for various increasing excitation voltages to include the negative electrostatic spring effect. To only show the increase in mechanical sensitivity, each $\mathrm{TF}$ is normalized with the additional electrical gain $\left(\sim A_{\mathrm{ex}}^{2}\right)$ 


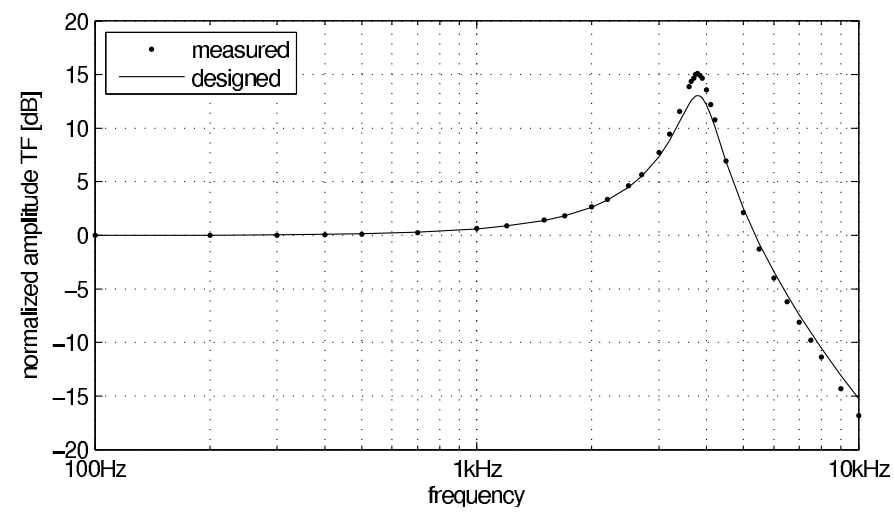

Figure 11: The measured and expected (designed) normalized mechanical amplitude transfer function.

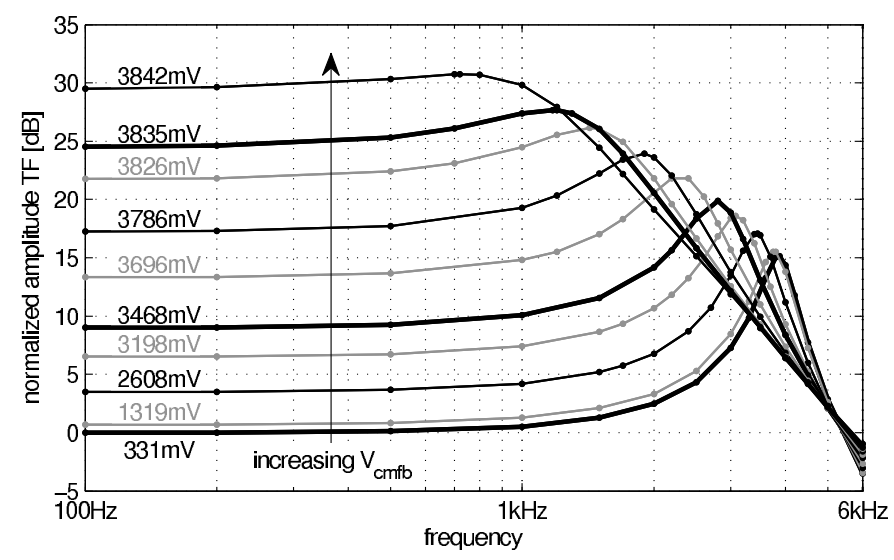

Figure 12: The normalized mechanical amplitude transfer function for an increasing voltage over the sense capacitors (corresponding to stronger negative springs). The corresponding voltage over the sense capacitors $V_{\mathrm{cmfb}, \mathrm{rms}}$ is annotated for each curve.

and the result is shown in Fig. 12. The corresponding rootmean-square (rms) value of the voltage over the sense capacitors $\left(V_{\mathrm{cmfb}}\right)$ is also given in the figure. We see that increasing $\sim A_{\mathrm{ex}}^{2}$ indeed leads to an increased mechanical sensitivity as predicted by Eq. (2) and (5). However, the voltage over the sense capacitors can not exceed the pull-in voltage. The measured pull-in voltage for this die is $3862 \mathrm{mV}$ (this is approximately $200 \mathrm{mV}$ higher than the expected value (Table 1). The closer $V_{\mathrm{cmfb}}$ gets to this pull-in voltage, the higher the corresponding mechanical gain increment will be.

Fig. 12 also shows that $f_{0}$ decreases as $V_{\mathrm{cmfb}}$ increases. This is also expected as $f_{0}$ is proportional to $\sqrt{k_{\text {net }} / m}$. Additionally, also the Q-factor of the mechanical transfer function decreases for increasing $V_{\mathrm{cmfb}}$ as it is equal to $\sqrt{m \cdot k_{\text {net }}} / b$. As $V_{\text {cmfb }}$ increases, the capacitor gap decreases and as result also the film squeeze damping increases which will further reduce the Q-factor.

The next measurements are performed on the closed loop force-feedback operation of the complete accelerometer system. First, the accelerometer is tested by turning it around in the gravitation field. The resulting output voltage $\left(V_{\text {out }}\right)$ is shown in Fig. 13. This shows that the input range is at least $\pm 1 \mathrm{~g}$ and

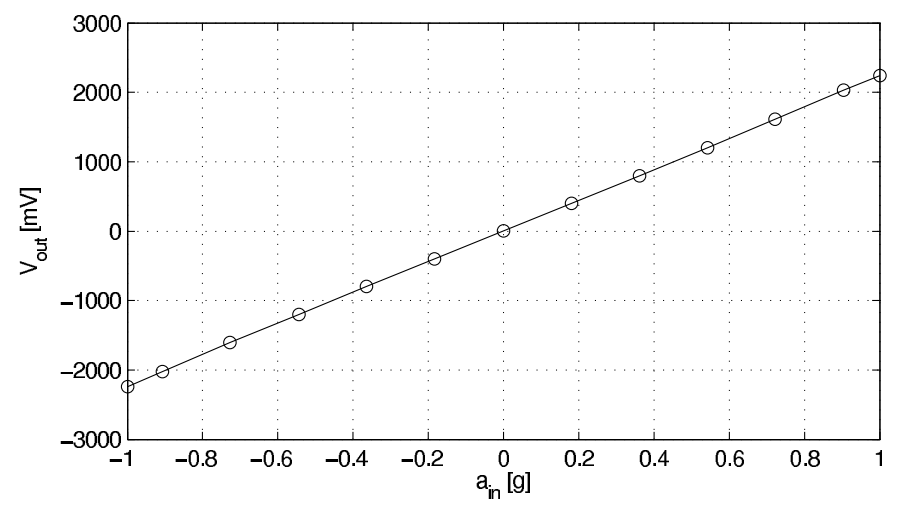

Figure 13: The output voltage vs the applied acceleration for the closed loop force-feedback operation of the complete accelerometer system.

the output has a conversion ratio of $2.25 \mathrm{~V} / \mathrm{g}$. This is close to the expected value of $1.8 \mathrm{~V} / \mathrm{g}$ given by equation (10) (25\% increase). The non-linearity of the curve is $\pm 0.7 \%$. This is within the specifications of the ADC used to digitize $V_{\text {out }}$.

Fig. 14 shows the measured output spectrum for a set of $A_{\text {ex }}(2,4$ and $6 \mathrm{~V})$ when no acceleration is acting upon the accelerometer (the corresponding $V_{\mathrm{cmfb}, \mathrm{rms}}$ are shown in the legend). This measurement is done by filtering $V_{\text {out }}$ with a $3^{\text {rd }}$ order Butterworth lowpass filter $(7.5 \mathrm{kHz}$ bandwidth) and sampling the result at $100 \mathrm{kHz}$. The measured voltage is then converted into a $g$ unit. As expected, the residual noise is reduced when the excitation voltage is increased due to the increase in electrical as well as mechanical gain. Small peaks around the mechanical resonance frequency $( \pm 3.9 \mathrm{kHz})$ are visible. For higher excitation voltages, this peak again moves toward lower frequencies, as was already highlighted when discussing Fig. 12. The spectrum also shows that a bandwidth of at least $200 \mathrm{~Hz}$ is reached for the different excitation voltages. This bandwidth can be further extended, as noted in Section 3.4, by using a more complex loop filter with improved frequency compensation to limit the influence of the mechanical transfer on the stability. For higher excitation voltages, a residual error tone at $50 \mathrm{~Hz}$ becomes visible.

Fig. 15 shows the residual noise floor in $[\mu g / \sqrt{\mathrm{Hz}}]$ versus the voltage over the sense capacitors $\left(V_{\mathrm{cmfb}, \mathrm{rms}}\right)$, measured on two MEMS dies. The measured noise density is almost completely the same for both dies. For lower voltages, the electrical noise of the readout circuit is clearly dominant. At higher voltages, a minimum noise floor of $4.1 \mu \mathrm{g} / \sqrt{\mathrm{Hz}}$ is reached at $V_{\mathrm{cmfb}}=3780 \mathrm{mV}\left(A_{\mathrm{ex}}=6125 \mathrm{mV}\right)$. This is between the expected values of $3.2 \mu \mathrm{g} / \sqrt{\mathrm{Hz}}$ (zero position) and $4.25 \mu \mathrm{g} / \sqrt{\mathrm{Hz}}$ (pull-in point). From this we can conclude that the measured noise floor corresponds to the mechanical noise floor.

With this, an important element has been demonstrated through the PCB-prototype: the dual-mass accelerometer with increased sensitivity leads to a much smaller input-referred contribution of the electrical noise. This element is quite favorable for applying these methods in an integrated circuit implementation. Such an integrated implementation could be based on circuits presented in [13], because the core of the readout circuits of the PCB-prototype largely emulates the same functionality 


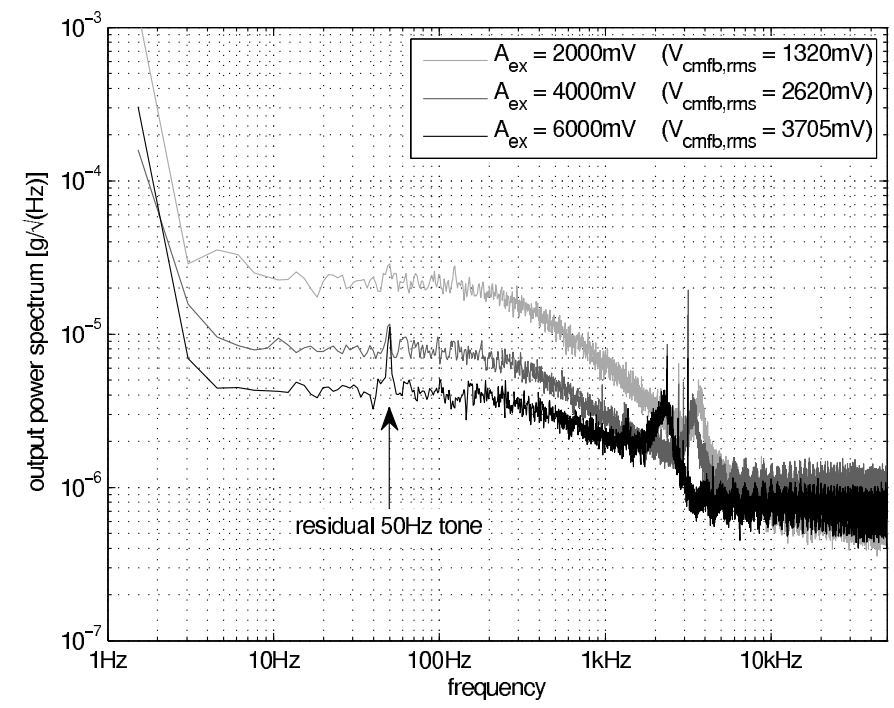

Figure 14: The measured output power spectrum for different excitation voltages (semi-overlapping 64K-points Hanning window over 1M sample points).

(see Section 3.2). It is then clear that the relaxed noise requirements on the readout circuits are expected to be quite beneficial for reducing the power consumption of these circuits. However, this element cannot be verified experimentally with the current PCB-prototype which is based on off-the-shelf components with $\pm 15 \mathrm{~V}$ supply.

Recently published state of the art capacitive readout accelerometers show an accuracy that goes below $\mu g$-resolution $[4,15,23]$. These accelerometers are made with more advanced MEMS processes which allows them to increase the proof mass and sense capacitors. This leads to increased mechanical sensitivity and a substantially lowered mechanical noise floor. The process used for this prototype is a commercially available surface-micromachined process with modest characteristics and consequently only a modest mechanical noise floor. However, the new dual-mass accelerometer system can also be implemented with these more advanced processes. Moreover, the increased mechanical and electrical sensitivity is an efficient way to reduce the influence of the electronic readout noise and attain the lowered mechanical noise floor limit for the overall accelerometer.

\section{Conclusion}

A new dual-mass accelerometer system is presented. Readout through the parallel-plate sense capacitors allows operation near pull-in, which increases the electrical and mechanical sensitivity. A readout circuit is presented which combines charge controlled common-mode stabilization with a differential-mode operated in voltage control. This results in a negative spring effect to compensate the mechanical spring while also reducing the sense capacitor gap. As a proof of concept, the new mechanical structure was fabricated and the electronic readout was implemented on a PCB. This prototype shows the feasibility of the new accelerometer concept, as well as it's best assets which

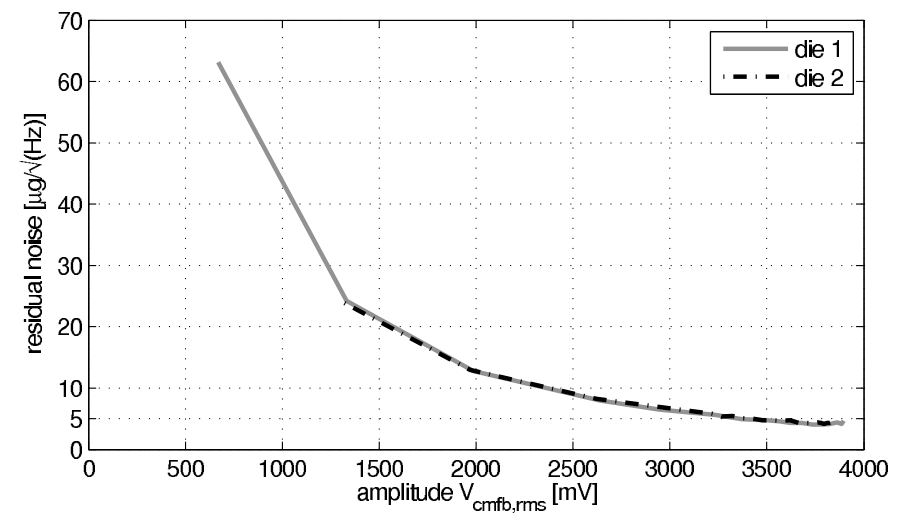

Figure 15: The measured noise spectral density for an increasing voltage over the sense capacitors $\left(V_{\mathrm{cmfb}}\right)$.

are an increased mechanical and electrical sensitivity. Measurements on this prototype show it achieves an accuracy of $4.1 \mu \mathrm{g} / \sqrt{\mathrm{Hz}}$ over an input range of at least $\pm 1 \mathrm{~g}$ and has a nonoptimized bandwidth of $200 \mathrm{~Hz}$.

\section{Acknowledgments}

This work was supported by the Special Research Fund (BOF) of Ghent University.

\section{References}

\section{References}

[1] S. D. Senturia, Microsystem design, Kluwer Academic Publishers, Norwell, MA, USA, 2001.

[2] N. Yazdi, F. Ayazi, K. Najafi, Micromachined inertial sensors, Proc. IEEE 86 (8) (1998) 1640-1659.

[3] G. Krishnan, C. Kshirsagar, K. Ananthasuresh, N. Bhat, Micromachined high-resolution accelerometers, J. Indian I. Sci. 87 (2007) 333-61.

[4] H. Kulah, J. Chae, N. Yazdi, K. Najafi, Noise analysis and characterization of a sigma-delta/capacitive microaccelerometer, IEEE J. Solid-State Circ. 41 (2) (2006) 352-361.

[5] M. Handtmann, R. Aigner, A. Meckes, G. Wachutka, Sensitivity enhancement of MEMS inertial sensors using negative springs and active control, Sens. Actuators A 97-8 (2002) 153-160.

[6] C. Acar, A. Shkel, Structurally decoupled micromachined gyroscopes with post-release capacitance enhancement, J. Micromech. Microeng. 15 (5) (2005) 1092-1101.

[7] K. Han, Y. Cho, Self-balanced navigation-grade capacitive microaccelerometers using branched finger electrodes and their performance for varying sense voltage and pressure, J. of Microelectromech. S. 12 (1) (2003) 11-20.

[8] P. Woestyn, J. Raman, P. Rombouts, L. Weyten, P. De Baets, A dual-mass capacitive-readout accelerometer operated near pull-in, in: 53rd IEEE Int. Midwest Symp. Circ. Syst. (MWSCAS 2010), 2010, pp. 1185-8.

[9] L. Castaner, J. Pons, R. Nadal-Guardia, A. Rodriguez, Analysis of the extended operation range of electrostatic actuators by current-pulse drive, Sens. Actuators A 90 (3) (2001) 181-190.

[10] J. Seeger, B. Boser, Charge control of parallel-plate, electrostatic actuators and the tip-in instability, J. of Microelectromech. S. 12 (5) (2003) $656-671$.

[11] G. Langfelder, T. Frizzi, A. Longoni, A. Tocchio, D. Manelli, E. Lasalandra, Readout of MEMS capacitive sensors beyond the condition of pull-in instability, Sens. Actuators A 167 (2, SI) (2011) 374-384.

[12] C. Lu, M. Lemkin, B. Boser, A monolithic surface micromachined accelerometer with digital output, IEEE J. Solid-State Circ. 30 (12) (1995) $1367-1373$ 
[13] M. Lemkin, B. Boser, A three-axis micromachined accelerometer with a CMOS position-sense interface and digital offset-trim electronics, IEEE J. Solid-State Circ. 34 (4) (1999) 456-468.

[14] Y. Dong, M. Kraft, C. Gollasch, W. Redman-White, A high-performance accelerometer with a fifth-order sigma-delta modulator, J. Micromech. Microeng. 15 (7, SI) (2005) S22-S29.

[15] R. Abdolvand, B. V. Amini, F. Ayazi, Sub-micro-gravity in-plane accelerometers with reduced capacitive gaps and extra seismic mass, J. of Microelectromech. S. 16 (5) (2007) 1036-1043.

[16] V. Petkov, B. Boser, High-order electromechanical Sigma Delta modulation in micromachined inertial sensors, IEEE T. Circuits-I 53 (5) (2006) 1016-1022.

[17] J. Raman, P. Rombouts, L. Weyten, An Unconstrained Architecture for Systematic Design of Higher Order Sigma Delta Force-Feedback Loops, IEEE T. Circuits-I 55 (6) (2008) 1601-1614.

[18] J. Raman, E. Cretu, P. Rombouts, L. Weyten, A Closed-Loop Digitally Controlled MEMS Gyroscope With Unconstrained Sigma-Delta ForceFeedback, IEEE Sens. J. 9 (3) (2009) 297-305.

[19] R. Legtenberg, A. Groeneveld, M. Elwenspoek, Comb-drive actuators for large displacements, J. Micromech. Microeng. 6 (3) (1996) 320-329.

[20] M. Bao, H. Yang, Squeeze film air damping in MEMS, Sens. Actuators A 136 (1) (2007) 3-27.

[21] T. Veijola, A. Pursula, P. Raback, Extending the validity of squeezed-film damper models with elongations of surface dimensions, J. Micromech. Microeng. 15 (9) (2005) 1624-1636.

[22] T. Gabrielson, Mechanical-thermal noise in micromachined acoustic and vibration sensors, IEEE T. Electron Dev. 40 (5) (1993) 903-909.

[23] J. Chae, H. Kulah, K. Najafi, An in-plane high-sensitivity, low-noise micro-g silicon accelerometer with CMOS readout circuitry, J. of Microelectromech. S. 13 (4) (2004) 628-635. 